Service social

\title{
Adolescents Parents Communication Couples - comment mieux comprendre..., par Helmi Farid, Montréal, Les éditions Agence d'Arc inc., 1988, 160 pages.
}

\section{Marie-Christine Saint-Jacques}

Volume 37, numéro 3, 1988

Index analytique 1978-1988

URI : https://id.erudit.org/iderudit/706416ar

DOI : https://doi.org/10.7202/706416ar

Aller au sommaire du numéro

Éditeur(s)

École de service social de l'Université Laval

ISSN

1708-1734 (numérique)

Découvrir la revue

Citer ce compte rendu

Saint-Jacques, M.-C. (1988). Compte rendu de [Adolescents Parents Communication Couples - comment mieux comprendre..., par Helmi Farid, Montréal, Les éditions Agence d'Arc inc., 1988, 160 pages.] Service social, 37(3), 467-469. https://doi.org/10.7202/706416ar d'utilisation que vous pouvez consulter en ligne.

https://apropos.erudit.org/fr/usagers/politique-dutilisation/ 
donc une mise en garde : "Le soin des malades, des handicapés et des personnes âgées était autrefois une responsabilité familiale, puis le flambeau est passé à l'État; cela est devenu une responsabilité gouvernementale. II est impensable de repasser, tel quel, le flambeau aux familles et aux individus... Les réseaux "naturels " sont déjà essoufflés dans bien des $\operatorname{cas}^{13}$ ".

Centre de recherche sur les services communautaires,

Jacques Ror

Université Laval.

\section{Références}

${ }^{1}$ Extraits du volume Le sort de la culture, Éditions de l'Hexagone, publiés dans Le Devoir, 19 décembre 1987, p. D1.

${ }^{2}$ Andrée Fortin et al., Histoires de familles et de réseaux, p. 87-88.

3 Ibid., p. 217.

4 Ibid., p. 16.

5 Ibid., p. 195.

6 Ibid., p. 147.

${ }^{7}$ Propos recueillis dans Le Soleil du 5 décembre 1987, p. A-17, suite à une conférence donnée à Paris et intitulée " La famille et la culture nord-américaine sous le choc de la modernité ".

${ }^{8}$ Andrée Fortin et al., op. cit., p. 66 et 175.

9 Ibid., p. 177.

${ }^{10}$ Commission d'Enquête sur les Services de Santé et les Services Sociaux, Dossier "personnes âgées ", programme de consultation d'experts, août 1987, p. 27.

11 Ibid., p. 57-58.

12 Ibid., p. 26.

${ }^{13}$ Andrée Fortin et al., op. cit., p. 221.

Adolescents Parents Communication Couples - comment mieux comprendre..., par Helmi FARID, Montréal, Les éditions Agence d'Arc inc., 1988, 160 pages.

Disons d'emblée de cet ouvrage qu'il s'adresse aux parents d'adolescents. II ne s'agit donc pas d'un livre spécialisé, mais plutôt d'un ouvrage que l'auteur a voulu rendre accessible à tous.

Ce livre, divisé en deux parties, traite dans un premier temps de différents thèmes propres à l'adolescence. Ainsi sont abordées les questions suivantes : corps et sexualité, groupe et amitié, école, autorité parentale, attitudes face aux mass média et à la société actuelle, tabac, alcool et drogue, suicide et, finalement, fugue. Chacune de ces questions fait l'objet d'un chapitre auquel l'auteur a annexé les résultats d'une enquête qu'il a menée auprès de 1932 élèves du secondaire. 
La seconde partie aborde plus spécifiquement la notion de communication à travers divers thèmes : le couple, le dialogue avec les adolescents, l'éducation sexuelle, la sexualité des parents, Cette partie se termine par un chapitre portant sur l'adolescent face à la séparation ou au divorce de ses parents.

Les choix idéologiques faits dans la première partie ont soulevé en nous certaines interrogations. En effet, l'auteur semble faire une lecture très traditionnelle de la famille qui, à notre avis, ne tient pas compte de l'évolution des valeurs des Québécois. Ainsi, à titre d'exemple, l'auteur souligne à plusieurs reprises l'importance des rôles distincts du père et de la mère, notamment au plan de l'autorité et de l'affection :

Le père est, dans l'esprit de l'enfant, le détenteur de l'autorité comme la mère est la dispensatrice de l'affection; mais cette distinction ne doit pas être prise à la lettre. L'autorité n'est pas interdite à la mère à condition qu'elle ne contrarie pas celle du père. (p. 43)

Voici une affirmation qui risque d'en surprendre plusieurs. Dans un ouvrage si récent et à portée éducative, il est assez étonnant de retrouver des principes qui sont à notre avis un peu dépassés. N'y aurait-il pas eu lieu d'insister sur la notion de coalition parentale par exemple ? Cette première partie prend aussi un ton inquiétant lorsque l'auteur aborde des questions comme le groupe d'amis, la masturbation, etc., jugeant "anormaux " certaines attitudes ou comportements que nous retrouvons fréquemment. Finalement, ce qui fait peut-être le plus problème, c'est qu'on aborde un très grand nombre de thèmes touchant l'adolescence sans les développer suffisamment ou en les fondant sur des valeurs que nous jugeons périmées. Les affirmations de l'auteur ne sont pas nécessairement fausses mais auraient nécessité plus de nuances. Ceci s'explique peut-être par le fait que la majorité des ouvrages de référence utilisés par l'auteur sont de sources françaises (et de ce fait, peut-être moins adaptés au contexte québécois) et que plus de la moitié d'entre eux ont été publiés avant les années quatre-vingt.

Enfin, tel que déjà mentionné, l'auteur complète chacun des thèmes par les réponses qu'ont fournies, sur ces sujets, les adolescents ayant participé à son enquête. Administrées à un échantillon représentatif de la population à l'étude, ces questions dressent un portrait des attentes et des sentiments des adolescents. Nous nous interrogeons cependant sur la validité de certaines des échelles d'attitudes utilisées. En effet, à quelques reprises, les questions proposent des choix de réponses qui nous semblent orientés en faveur d'une attitude précise.

Quoi qu'il en soit, cette façon de faire nous est apparue avantageusement complémentaire puisqu'elle donne une voix aux adolescents qui ont ainsi la chance d'exprimer leur point de vue. Ceci ne pourra qu'aider les parents à se faire une idée plus juste de la réalité. Il aurait été par ailleurs intéressant et ce, malgré les réserves émises par l'auteur, que ce dernier, à travers la rédaction des chapitres, fasse le lien avec les données recueillies auprès des jeunes. Nous ne croyons pas que ce procédé ait empêché le lecteur de tirer ses propres conclusions, au contraire.

La seconde partie du livre aborde principalement le thème de la communication et est de facture différente. L'auteur y présente moins d'idées 
mais les développe pius longuement, apportant les nuances nécessaires. Cette partie a aussi la qualité de bien expliquer au lecteur ce qu'est la communication et comment l'utiliser efficacement pour résoudre des difficultés, qu'elles soient d'ordre conjugal ou parental. Trop de livres ont présenté aux parents la communication comme étant " la solution "à tous les maux sans en expliquer les fondements et la façon d'améliorer cette communication au sein de la famille. L'auteur a su, avec habileté, soulever différentes situations auxquelles peuvent être confrontés les parents (par exemple l'adolescent qui consomme de la drogue ou qui veut abandonner l'école) et démontrer comment dialoguer afin d'en arriver à une solution satisfaisante.

Dans cette partie, la seule ombre au tableau a trait au modèle que procurent les parents aux enfants. L'auteur affirme en effet "qu'un modèle parental rassurant et attirant pourrait éviter toute forme ultérieure d'homosexualité chez l'adolescent et rendra l'hétérosexualité son but primordial " (p. 118). Loin de nier l'importance pour le développement futur de l'enfant de l'image d'adultes que lui procurent ses parents, nous croyons que cette affirmation relève plus de mythes et de préjugés que de la réalité. En effet, combien de parents se sont culpabilisés et ont remis en question l'éducation donnée à leurs enfants parce que l'un d'entre eux se révélait homosexuel. On peut aussi se demander pourquoi l'hétérosexualité devrait-elle être l'idéal recherché par tous.

Finalement, nous avons trouvé particulièrement intéressant le dernier chapitre traitant de I'adolescent face à la rupture parentale. On y retrouve deux idées maîtresses : une première voulant que des relations tendues entre ex-conjoints accroissent les effets du divorce, une seconde soulignant l'importance du maintien du lien parental après une séparation ou un divorce (idée dont on entendra de plus en plus parler dans les années à venir).

Somme toute, il s'agit d'un livre d'introduction qui aborde diverses questions touchant l'adolescence, la famille et le couple. Tout en tenant compte des réserves émises, cet ouvrage demeure un document intéressant pour sa seconde partie.

Marie-Christine SAINT-JACQUES

Étudiante à la maîtrise,

École de service social,

Université Laval.

L'enfant en famille d'accueil, par Denise Bourgault et Françoise DE LA HARPE, Saint-Hyacinthe, ÉDISEM, 1988, 120 pages.

Voici un volume qui cherche sans prétention mais avec beaucoup d'efficacité à informer et à guider celles et ceux qui ont à intervenir dans le cadre d'un placement d'enfant en famille d'accueil. Utilisant à la fois des données de l'expérience et des connaissances théoriques tirées des champs les plus pertinents à ce secteur d'intervention, les auteures tentent de cerner les conditions qui permettront au placement familial en contexte volontaire d'avoir des vertus thérapeutiques pour l'enfant et sa famille. 